\title{
Radiation and Mass Transfer Effects on MHD Free Convective Dissipative Fluid in the Presence of Heat Source/Sink
}

\author{
S. Suneetha ${ }^{1}$, N. Bhaskar Reddy ${ }^{2}$ and V. Ramachandra Prasad ${ }^{3}$ \\ ${ }^{1}$ Assistant Professor, Department of Mathematics, Yogi vemana University College of Engineering, Proddutor, \\ Kadapa, A.P., Inda. \\ ${ }^{2}$ Professor, Department of Mathematics, Sri Venkateswara University, Tirupati - 517502, A.P., INDIA, \\ ${ }^{3}$ Associate Professor, Department of Mathematics, MITS, Madanapalle,A.P,INDIA, \\ Email: ${ }^{1}$ suneetha_svu@yahoo.co.in_2nbrsvu@yahoo.co.in ${ }^{3}$ rcpmaths@mits.ac.in
}

(Received November 01, 2008; accepted January 23, 2010)

\begin{abstract}
Thermal radiation effects on MHD flow past an impulsively started vertical plate in the presence of heat source/sink is investigated, by taking into account the heat due to viscous dissipation. The governing boundary layer equations of the flow field are solved by an implicit finite difference method of Crank-Nicholson type. A parametric study is performed to illustrate the influence of radiation parameter, magnetic parameter, Grashof number, Prandtl number, Eckert number on the velocity, temperature and concentration profiles. Also, the local and average skin-friction, Nusselt number and Sherwood number are presented graphically. The numerical results reveal that the radiation induces a rise in both the velocity and temperature, and a decrease in the concentration. Also with an increase in the heat absorption/generation parameter the velocity increases whereas the temperature decreases. The model finds applications in solar energy collection systems, geophysics and astrophysics, aero space and also in the design of high temperature chemical process systems.
\end{abstract}

Keywords: Radiation, MHD, viscous dissipation, heat generation/absorption.

\section{INTRODUCTION}

In the processes involving high temperatures, the radiation heat transfer in combination with conduction, convection and also mass transfer plays very important role in the design of pertinent equipments in the areas such as nuclear power plants, gas turbines and the various propulsion devices for air craft, missiles, satellites and space vehicles. Chamkha et al. ( 2001) studied the radiation effects on the free convection flow past a semi-infinite vertical plate with mass transfer. Muthucumaraswamy and Kumar Senthil (2004) investigated the heat and mass transfer effect on moving vertical plate in the presence of thermal radiation. Ramachandra Prasad et al. (2007) considered the radiation and mass transfer effects on twodimensional flow past an impulsively started isothermal vertical plate. The interaction of radiation with hydromagnetic flow has become industrially more prominent in the processes wherever high temperatures occur. Chaudhary et al. (2006) studied the radiation effect with simultaneous thermal and mass diffusion in MHD mixed convection flow from a vertical surface. Ramachandra Prasad et al. (2006) studied the transient radiative hydromagnetic free convection flow past an impulsively started vertical plate with uniform heat and mass flux.

The heat source/sink effects in thermal convection, are significant where there may exist a high temperature differences between the surface (e.g. space craft body) and the ambient fluid. Heat generation is also important in the context of exothermic or endothermic chemical reactions Chamkha (2000) investigated thermal radiation and buoyancy effects on hydromagnetic flow over an accelerating permeable surface with heat source or sink. Ramachandra Prasad et al. (2006) analysed the radiation effects on an unsteady two dimensional hydromagnetic free convective boundary layer flow of a viscous incompressible fluid past a semi-infinite vertical plate with mass transfer in the presence of heat source or sink.

Viscous mechanical dissipation effects are important in geophysical flows and also in certain industrial operations and are usually characterized by the Eckert number. Mahajan and Gebhart (1989) reported the influence of viscous heating dissipation effects in natural convective flows, showing that the heat transfer rates are reduced by an increase in the dissipation 
parameter. The influence of viscous dissipation and radiation on unsteady MHD free-convection flow past an infinite heated vertical plate in a porous medium with time-dependent suction was studied by IsraelCookey et al. (2003). Ramachandra Prasad and Bhaskar Reddy (2007) presented radiation and mass transfer effects on an unsteady MHD free convection flow past a heated vertical plate with viscous dissipation. Zueco (2007) used Network Simulation method [NSM] to study the effects of viscous dissipation and radiation on unsteady MHD free convection flow past a vertical porous plate. Vasu et al. (2011) presented radiation and mass transfer effects on transient free convection flow of a dissipative fluid past semi-infinite vertical plate with uniform heat and mass flux.

However, the interaction of radiation with mass transfer of a hydro magnetic, dissipative fluid in the presence of heat source/sink has received little attention. Hence, the present study is attempted.

\section{Mathematical ANAlysiS}

An unsteady two-dimensional laminar natural convection flow of a viscous, incompressible, electrically conducting, radiating, dissipative and optically thick fluid past an impulsively started semiinfinite vertical plate is considered. A temperature dependent heat source (or sink) is assumed to be present in the flow. The fluid is assumed to be gray, absorbingemitting but non-scattering. The $\mathrm{x}$-axis is taken along the plate in the upward direction and the y-axis is taken normal to it.. A uniform magnetic field is applied in the direction perpendicular to the plate. The transverse applied magnetic field and magnetic Reynolds number are assumed to be very small, so that the induced magnetic field is negligible. Then, in the absence of input electric field, under the usual Boussinesq's approximation, the governing boundary layer equations are

$$
\begin{aligned}
& \frac{\partial u}{\partial x}+\frac{\partial v}{\partial y}=0 \\
& \frac{\partial u}{\partial t^{\prime}}+u \frac{\partial u}{\partial x}+v \frac{\partial u}{\partial y}=g \beta\left(T^{\prime}-T_{\infty}^{\prime}\right) \\
& +g \beta^{*}\left(C^{\prime}-C_{\infty}^{\prime}\right)+v \frac{\partial^{2} u}{\partial y^{2}}-\frac{\sigma B_{0}^{2}}{\rho} u \\
& \frac{\partial T^{\prime}}{\partial t^{\prime}}+u \frac{\partial T^{\prime}}{\partial x}+v \frac{\partial T^{\prime}}{\partial y}=\alpha \frac{\partial^{2} T^{\prime}}{\partial y^{2}}- \\
& \frac{1}{\rho c_{p}} \frac{\partial q_{r}}{\partial y}+\frac{v}{c_{p}}\left(\frac{\partial u}{\partial y}\right)^{2}+\frac{Q_{0}}{\rho c}\left(T^{\prime}-T_{\infty}^{\prime}\right) \\
& \frac{\partial C^{\prime}}{\partial t^{\prime}}+u \frac{\partial C^{\prime}}{\partial x}+v \frac{\partial C^{\prime}}{\partial y}=D \frac{\partial^{2} C^{\prime}}{\partial y^{2}}
\end{aligned}
$$

The initial and boundary conditions are

$$
t^{\prime} \leq 0: \quad u=0, v=0, T^{\prime}=T_{\infty}^{\prime}, C^{\prime}=C_{\infty}^{\prime}
$$

$$
\begin{array}{ll}
t^{\prime}>0: \quad u=0, v=0, T^{\prime}=T_{w}^{\prime}, C^{\prime}=C_{w}^{\prime} & \text { at } y=0 \\
u=0, v=0, T^{\prime}=T_{\infty}^{\prime}, C^{\prime}=C_{\infty}^{\prime} & \text { at } x=0 \\
u \rightarrow 0, T^{\prime} \rightarrow T_{\infty}^{\prime}, C^{\prime} \rightarrow C_{\infty}^{\prime} \quad \text { as } y \rightarrow \infty &
\end{array}
$$

where $u, v$ are the velocity components in $x, y$ directions respectively, $t^{\prime}$ - the time, g - the acceleration due to gravity, $\beta$ - the volumetric coefficient of thermal expansion, $\beta^{*}$ - the volumetric coefficient of expansion with concentration, $T^{\prime}$ - the temperature of the fluid in the boundary layer, $C^{\prime}$ - the species concentration in the boundary layer, $v$ - the kinematic viscosity, $T_{w}^{\prime}$ - the wall temperature, $T_{\infty}^{\prime}$ - the free stream temperature far away from the plate, $C_{w}^{\prime}$ - the concentration at the plate, $C_{\infty}^{\prime}$ - the free stream concentration far away from the plate, $\sigma$ - the electrical conductivity, $B_{0}-$ the magnetic induction, $\rho$ - the density of the fluid, $\alpha$ - the thermal diffusivity, $c_{p}$ - the specific heat at constant pressure, $q_{r}$ - the radiation heat flux, $\mathrm{Q}_{0}-$ the heat generation/absorption and $D$ - the species diffusion coefficient.

Thermal radiation is assumed to be present in the form of a unidirectional flux in the y-direction i.e., $q_{r}$ (transverse to the vertical surface). By using the Rosseland approximation (Brewster, 1992), the radiative heat flux $q_{r}$ is given by

$q_{r}=-\frac{4 \sigma_{s}}{3 k_{e}} \frac{\partial T^{\prime 4}}{\partial y}$

where $\sigma_{s}$ is the Stefan-Boltzmann constant and $k_{e}$ the mean absorption coefficient.. If the temperature differences within the flow are sufficiently small, then Eq. (6) can be linearized by expanding $T^{\prime 4}$ into the Taylor series about $T_{\infty}^{\prime}$, which after neglecting higher order terms takes the form

$T^{\prime 4} \cong 4 T_{\infty}^{\prime 3} T^{\prime}-3 T_{\infty}^{\prime 4}$

In view of Eqs. (6) and (7), Eq. (3) reduces to

$$
\begin{aligned}
& \frac{\partial T^{\prime}}{\partial t^{\prime}}+u \frac{\partial T^{\prime}}{\partial x}+v \frac{\partial T^{\prime}}{\partial y}=\alpha \frac{\partial^{2} T^{\prime}}{\partial y^{2}}+ \\
& \frac{16 \sigma_{s} T_{\infty}^{\prime} 3}{3 k_{e} \rho c_{p}} \frac{\partial^{2} T^{\prime}}{\partial y^{2}}+\frac{v}{c_{p}}\left(\frac{\partial u}{\partial y}\right)^{2}+\frac{Q_{0}}{\rho c_{p}}\left(T^{\prime}-T_{\infty}^{\prime}\right)
\end{aligned}
$$

Using the following non-dimensional quantities

$$
\begin{aligned}
& X=\frac{x}{L}, Y=\frac{y G r^{1 / 4}}{L}, t^{\prime}=\frac{t L^{2}}{v} G r^{-1 / 2}, U=\frac{u L G r^{-1 / 2}}{v} \\
& G r=\frac{g \beta L^{3}\left(T_{w}^{\prime}-T_{\infty}^{\prime}\right)}{v^{2}}, \varphi=\frac{Q_{0} L^{2}}{\rho c_{p} v G r / 2},
\end{aligned}
$$




$$
\begin{aligned}
& T=\frac{T^{\prime}-T_{\infty}^{\prime}}{T_{w}^{\prime}-T_{\infty}^{\prime}}, C=\frac{C^{\prime}-C_{\infty}^{\prime}}{C_{w}^{\prime}-C_{\infty}^{\prime}}, \operatorname{Pr}=\frac{v}{\alpha}, \\
& F=\frac{k_{e} k}{4 \sigma_{s} T_{\infty}^{\prime 3}}, \quad S c=\frac{v}{D} \\
& E c=\frac{u_{O}^{2}}{c_{p}\left(T_{w}^{\prime}-T_{\infty}^{\prime}\right)}, \quad N=\frac{\beta^{*}\left(C_{w}^{\prime}-C_{\infty}^{\prime}\right)}{\beta\left(T_{w}^{\prime}-T_{\infty}^{\prime}\right)}, \\
& M=\frac{\sigma B_{0}^{2}}{u_{0}^{2}}, V=\frac{v L G r^{-1 / 4}}{v}
\end{aligned}
$$

where $\mathrm{L}$ is the characteristic length of the plate and $\mathrm{k}$ is the thermal conductivity. Eqs. (1), (2), (8) and (4) reduce to the following non-dimensional form

$$
\begin{aligned}
& \frac{\partial U}{\partial X}+\frac{\partial V}{\partial Y}=0 \\
& \frac{\partial U}{\partial t}+U \frac{\partial U}{\partial X}+V \frac{\partial U}{\partial Y}=\frac{\partial^{2} U}{\partial Y^{2}}+T+N C-M U \\
& \frac{\partial T}{\partial t}+U \frac{\partial T}{\partial X}+V \frac{\partial T}{\partial Y}=\frac{1}{\operatorname{Pr}}\left(1+\frac{4}{3 F}\right) \frac{\partial^{2} T}{\partial Y^{2}} \\
& +E c\left(\frac{\partial u}{\partial y}\right)+\varphi T \\
& \frac{\partial C}{\partial t}+U \frac{\partial C}{\partial X}+V \frac{\partial C}{\partial Y}=\frac{1}{S c} \frac{\partial^{2} C}{\partial Y^{2}}
\end{aligned}
$$

where, $G r, M, F, P r, E c, N, \varphi$ and $S c$ are thermal Grashof number, magnetic parameter, radiation parameter, Prandtl number, Eckert number, buoyancy parameter, the dimensionless heat generation/absorption coefficient and Schmidt number respectively. The corresponding initial and boundary conditions are

$$
\begin{aligned}
& t \leq 0: U=0, V=0, \quad T=0, C=0 \\
& t>0: U=0, V=0, T=1, C=1 \quad \text { at } \quad Y=0 \\
& U=0, V=0, T=0, C=0 \quad \text { at } \quad X=0 \\
& U \rightarrow 0, T \rightarrow 0, C \rightarrow 0 \quad \text { as } Y \rightarrow \infty
\end{aligned}
$$

For the type of flow under consideration, the local as well as average values of skin-friction, Nusselt number and Sherwood number are important, which in dimensionless form are given by

$$
\begin{aligned}
& \tau_{X}=\frac{\tau^{\prime}}{\rho u_{0}^{2}}=-\left(\frac{\partial U}{\partial Y}\right)_{Y=0} \\
& \bar{\tau}=-\int_{0}^{1}\left(\frac{\partial U}{\partial Y}\right)_{Y=0} d X \\
& N u_{X}=-X\left[\left(\frac{\partial T}{\partial Y}\right)_{Y=0} / T_{Y}=0\right] \\
& \overline{N u}=-\int_{0}^{1}\left[\left(\frac{\partial T}{\partial Y}\right)_{Y=0} / T_{Y}=0\right] d x
\end{aligned}
$$

$$
\begin{gathered}
S h_{X}=-X\left[\left(\frac{\partial C}{\partial Y}\right)_{Y=0} / C_{Y}=0\right] \\
\overline{S h}=-\int_{0}^{1}\left[\left(\frac{\partial C}{\partial Y}\right)_{Y=0} / C_{Y}=0\right] d x
\end{gathered}
$$

\section{NUMERICAL TECHNIQUE}

In order to solve the unsteady, non-linear, coupled Eqs. (10) - (13) under the conditions (14), an implicit finite difference scheme of Crank-Nicholson type has been employed. The region of integration is considered as a rectangle with sides $X_{\max }(=1)$ and $Y_{\max }(=14)$, where $Y_{\max }$ corresponds to $Y=\infty$, which lies very well outside the momentum, energy and concentration boundary layers. The maximum of $Y$ was chosen as 14 after some preliminary investigations so that the last two of the boundary conditions of Eq. (14) are satisfied. After experimenting with a few set of mesh sizes, the mesh sizes have been fixed at the level $\Delta X=0.05$, $\Delta Y=0.25$, and the time step $\Delta t=0.01$. in this case the special mesh sizes are reduced by $50 \%$ in the $\mathrm{Y}$ direction, the results differ in the fifth decimal place while the mesh sizes are reduced by $50 \%$ in X-direction or in both directions ; the results are comparable to three decimal places. Hence, the above mesh sizes have been considered as appropriate for calculation. The region to be examined in $(\mathrm{X}, \mathrm{Y}, \mathrm{t})$ space is covered by a rectilinear grid with sides parallel to axes with $\Delta \mathrm{X}, \Delta \mathrm{Y}$, and $\Delta \mathrm{t}$, as the grid spacing in $\mathrm{X}, \mathrm{Y}$ and $\mathrm{t}$ directions, respectively. An appropriate mesh sizes considered for the calculations are $\Delta X=0.05, \Delta Y=0.25$, and the time step $\Delta t=0.01$. The finite difference equations corresponding to the Eqs. (10)-(13) are considered. During any one time step, the coefficients appearing in finite difference equations are treated as constants. The finite difference equations at every internal nodal point on a particular i - level constitute a tri diagonal system of equations, which are solved by Thomas algorithm as discussed in Carnahan et al. (1969). Computations are carried out until the steady state is reached. The steadystate solution is assumed to have been reached, when the absolute difference between the values of velocity, temperature and concentration at two consecutive time steps is less than $10^{-5}$ at all grid points. The derivatives involved in Eqs. (15)-(17) are evaluated using fivepoint approximation formula and then the integrals are evaluated using Newton-Cotes closed integration formula.

The truncation error in the finite difference approximation is $O\left(\Delta t^{2}+\Delta Y^{2}+\Delta X\right)$ and it tends to zero as $\Delta t, \Delta Y$ and $\Delta X$ tend to zero. Hence the scheme is compatible. The finite difference scheme is unconditionally stable. Stability and compatibility ensure convergence.

\section{RESUlts AND Discussion}

Extensive computations have been performed for the effects of the controlling thermo fluid and hydrodynamic parameters on the dimensionless velocity, temperature and concentration, and also on the 
local and average skin frictions, Nusselt numbers and Sherwood numbers.

In order to ascertain the accuracy of the numerical results, the present study is compared with the previous study. The velocity profiles for $S c=0.3,0.6, P r=0.71$, $N=1.0, M=1.0, E c=0.001, F=10, \phi=0.0, X=1.0$ are compared with the available solution of Prasad et al. in Fig. 1. It is observed that the present results are in good agreement with that of Prasad et al. (2006).

The effects of the radiation parameter $F$, the Schmidt number $S c$, and the magnetic parameter $M$ on the transient velocity profiles are illustrated in Fig. 2. The effects of $F$, on the transient temperature profiles are displayed in Fig. 4. From Figs. 2 and 4, it can be seen that an increase in the thermal radiation parameter produces significant increases in the thermal condition of the fluid and its thermal boundary layer. Through the buoyancy effect this increase in the fluid temperature induces more flow in the boundary layer causing the velocity of fluid there to increase. In addition the hydrodynamic boundary layer thickness increases as a result of increasing $F$. Further it is observed that the time required to reach the steady state increases with the increase in $F$. Also, it is clear that as the magnetic field parameter $M$ increases; the Lorentz force, which opposes the flow also increases and leads to an enhanced deceleration of the flow. This result qualitatively agrees with the expectations, since the magnetic field exerts a retarding force on the free convection flow (Fig. 2). The presence of a heat source in the boundary layer generates energy, which causes the temperature of the fluid to increase. This increase in temperature produces an increase in the flow field due to the buoyancy effect. These behaviors are evident from Fig. 3.

The effect of foreign mass on the transient velocity and concentration profiles can be observed from Figs. 2 and 5 . It can be easily seen that an increase in the $S c$ leads to a decrease in the velocity and concentration in the presence of an aiding buoyancy force. The time required to reach the steady state increases with the increase in $S c$. The effect of buoyancy ratio parameter $N$ on the steady state velocity, temperature and concentration profiles is shown in Figs. 6-8. It is observed that as the aiding buoyancy force increases, the velocity increases, whereas the temperature and concentration decrease. Figs. 9 and 10 show the effects of $\mathrm{Pr}$ and $\mathrm{Ec}$ on the transient velocity and temperature distributions. Both velocity and temperatures decrease as Pr increases. This is in agreement with the physical fact that the thermal boundary layer thickness decreases with increasing Pr. The effect of Ec in the flow field is to increase the energy, yielding a greater fluid temperature and as a consequence greater buoyancy force. The increase in the buoyancy force due to increase in the dissipation parameter enhances the convective velocity. These effects of dissipation on the velocity field are also the consequence of the behavioral changes in the temperature.

The influences of $G r, G m$ and $N$ on the temperature are shown in the Fig. 11. The thermal Grashof number $G r$ signifies the relative effect of the thermal buoyancy force to the viscous hydrodynamic force in the boundary layer. The solutal Grashof number $\mathrm{Gm}$ defines the ratio of the species buoyancy force to the viscous hydrodynamic force. An increase in $\mathrm{Gr}$ or $\mathrm{Gm}$ induces a sizeable decrease in the temperature throughout the porous regime. The buoyancy forces depress the temperatures in the medium. It is also observed that as $N$ increases, the temperature and the thermal boundary layer thickness decrease. Figs. 12 and 13 illustrate the effects of $F, E c, S c, \phi, M$, and $N$ on the local and average skin-frictions. The local and average skin-frictions are found to decrease due to an increase in $\mathrm{M}$ or $S c$. An increase in $N$ or $\phi$ or $E c$ produces an increase in the local skin-friction. It is observed that the local and average skin-frictions increase as $F$ increases. Figs. 14 and 15 show the effect of radiation parameter $F$ on the local and average Nusselt numbers respectively. It is observed that the local and average Nusselt numbers decrease as $F$ increases. The effect of heat source/sink parameter $\phi$ on the local Nusselt number is shown in Fig. 16. It can be easily seen that an increase in $\phi$ leads to a decrease in the local Nusselt number. Figures 17 and 18 display the effect of $S c$ on the local and average Sherwood numbers respectively. It can be observed that as $S c$ increases the local and average Sherwood numbers increase.

\section{CONCLUSIONS}

The present study investigates the interaction of radiation with mass transfer of a hydromagnetic, dissipative fluid in the presence of heat source/sink. As the radiation parameter increases, both the velocity and temperature increase, whereas the concentration decreases. An increase in the magnetic parameter decelerates the velocity. A rise in dissipative heat induces a substantial rise in both velocity and temperature. As the heat absorbtion/generation parameter increases, the velocity increases whereas the temperature decreases. An increase in the Schmidt number leads to a decrease in the velocity and concentration.

\section{REFERENCES}

Brewster, M.Q. (1992). Thermal Radiative Transfer and properties. John Wiley \& Sons. Inc., New York.

Chaudary, R.C., K.S. Bhupendra and J.A. Kumar (2006). Radiation effect with simultaneous thermal and mass diffusion in MHD mixed convection flow from a vertical surface with ohmic heating. Romanian Journal of Physics 51(7-8), Bucharest, 715-727.

Chamkha, A.J., H.S. Takhar and V.M. Soundalgekar (2001). Radiation effects on a free convection flow past a semi-infinite vertical plate with mass transfer. Chem. Engg. J. 84, 335- 342.

Chamkha, A.J. (2000). Thermal radiation and buoyancy effects on hydromagnetic flow over an accelerating permeable surface with heat source or sink. Int .J. Heat Mass Transfer 38, 1699-1712. 
Carnahan, B., H.A. Luther and J.O. Willkes (1969). Applied Numerical Methods. John Wiley \& Sons, New York.

Israel-Cookey, C., A. Ogulu and V.M. Omubo-Pepple (2003), Influence of viscous dissipation on unsteady MHD free convection flow past an infinite vertical plate in porous medium with time dependent suction. Int. J. Heat Mass Transfer 46, 2305-2311.

Joaquin, Z.J. (2007). Network Simulation Method Applied to Radiation and Dissipation Effects on MHD Unsteady Free Convection over Vertical Porous plate. Appl. Math. Modelling 31, 20192033.

Mahajan, R.L. and B.B. Gebhart (1989), Viscous dissipation effects in buoyancy-induced flows. Int . J. Heat Mass Transfer 32(7), 1380-1382.

Muthukumaraswamy, R. and G. Kumar Senthil (2004). Heat and Mass transfer effect on moving vertical plate in the presence of thermal radiation. Theoret. Appl. Mech., 31-35.

Ramachandra Prasad, V., N. Bhaskar Reddy and R. Muthukumaraswamy (2007). Radiation and mass transfer effects on two-dimensional flow past an impulsively started isothermal vertical plate. Int. J. Thermal Sciences 46(12), 1251-1258.

Ramachandra Prasad, V., N. Bhaskar Reddy and R. Muthucumaraswamy (2006). Transient Radiative hydromagnetic free convection flow past an impulsively started vertical plate with uniform heat and mass flux. Theoretical Applied Mechanics 33(1), 31- 63 .

Ramachandra Prasad, V., N. Bhaskar Reddy and R. Muthukumaraswamy (2006). Finite difference analysis of Radiation and Mass transfer effects on MHD free convection flow past a vertical plate in the presence of heat source/sink. Int. Review of Pure and Applied Mathematics 2(2), 141-160.

Ramachandra Prasad, V. and N. Bhaskar Reddy (2007). Radiation and mass transfer effects on unsteady MHD free convection flow past a heated vertical plate in a porous medium with viscous dissipation. Theoretical Applied Mechanics 34(2), 135-160.

Vasu, B., V. Ramachandra Prasad and N. Bhaskar Reddy (2011). Radiation and mass transfer effects on transient free convection flow of a dissipative fluid past semi-infinite vertical plate with uniform heat and mass flux, to appear in JAFM 4(1), 2011.

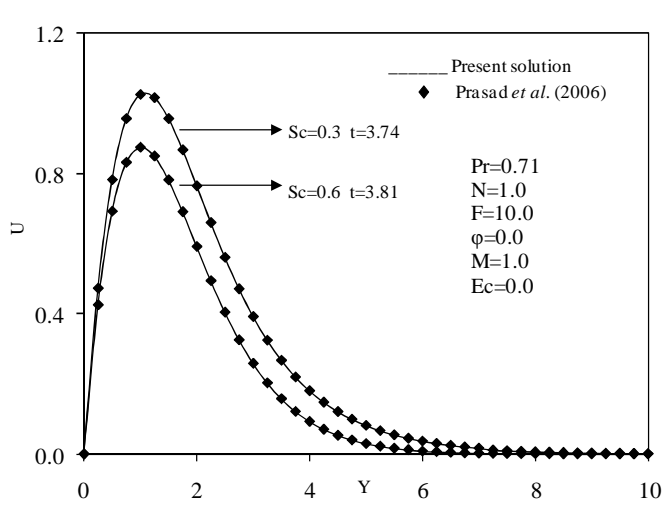

Fig. 1. Comparison of velocity profiles at $\mathrm{X}=1.0$.

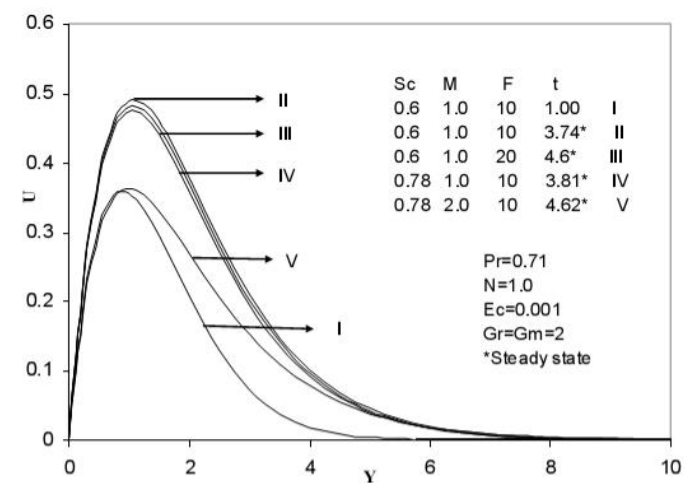

Fig. 2. Transient velocity profiles for different Sc $\mathrm{M}$ and $\mathrm{F}$.

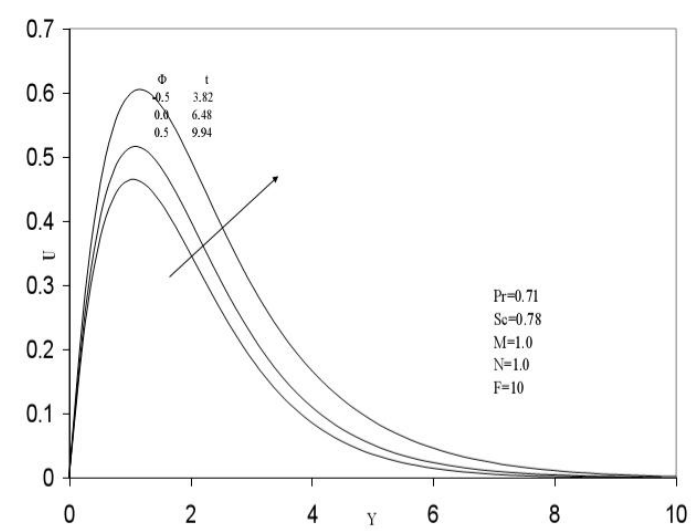

Fig. 3. Steady state velocity profiles for different $\Phi$.

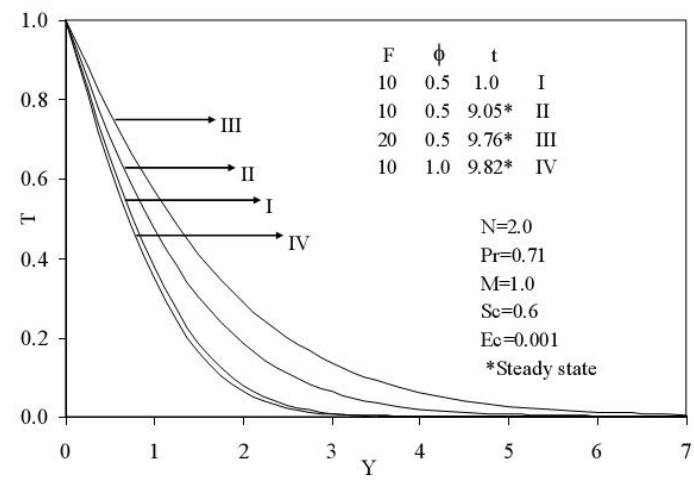

Fig. 4. Transient temperature profiles for different $\mathrm{F}, \phi$. 


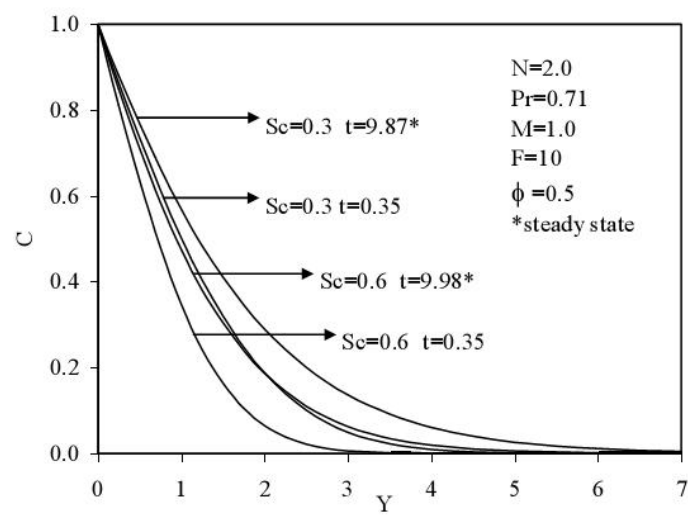

Fig.5. Transient concentration profiles for different Sc.

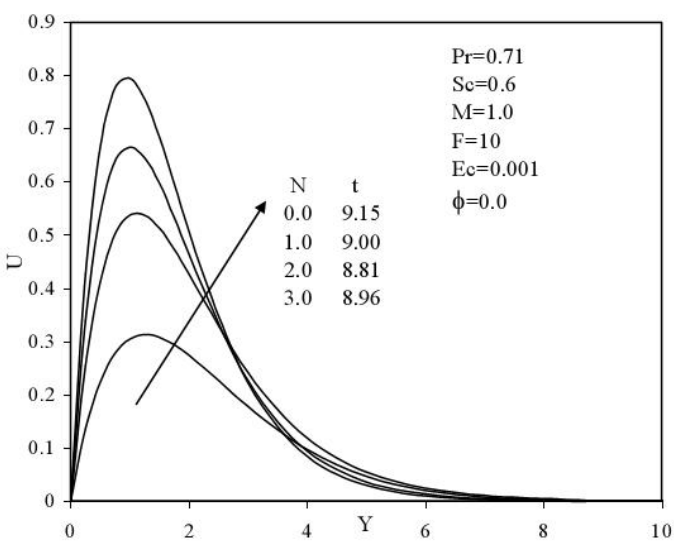

Fig.6. Effect of $\mathrm{N}$ on steady state velocity profiles.

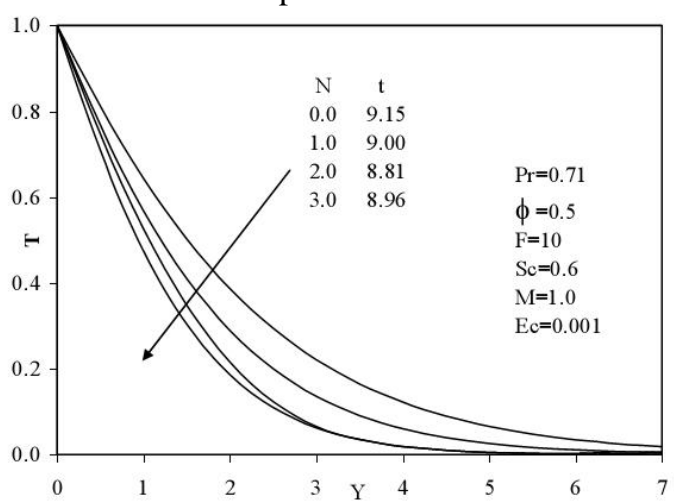

Fig.7. Effect of $\mathrm{N}$ on steady state temperature profiles.

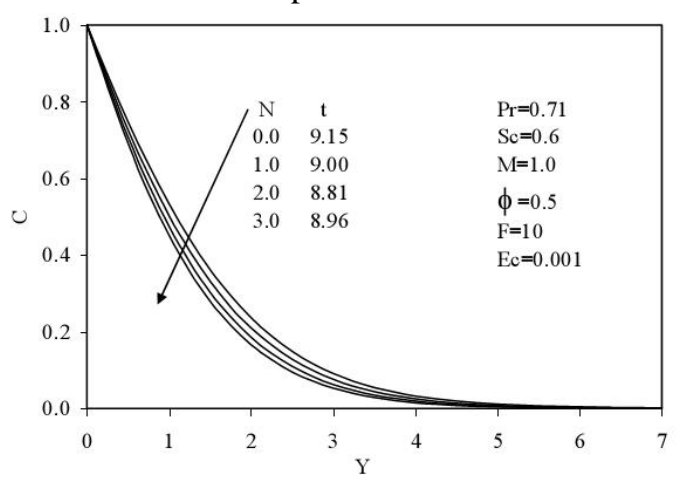

Fig. 8. Effect of $\mathrm{N}$ on steady state concentration profiles.

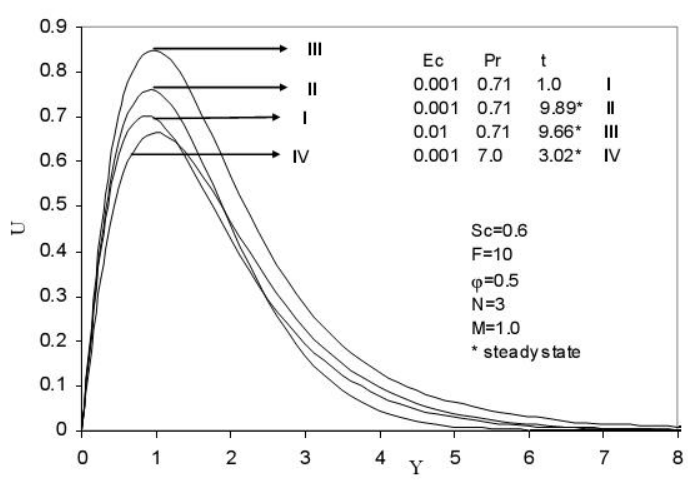

Fig. 9. Transient velocity profiles for different values of Ec and Pr.

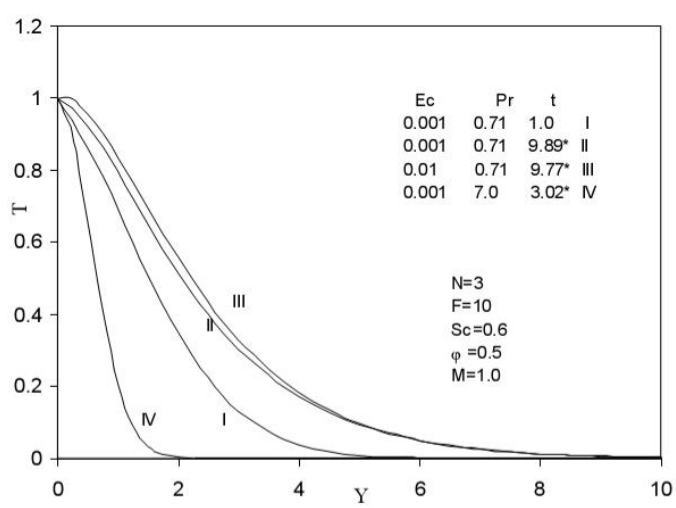

Fig. 10. Transient temperature profiles for different values of Ec and Pr.

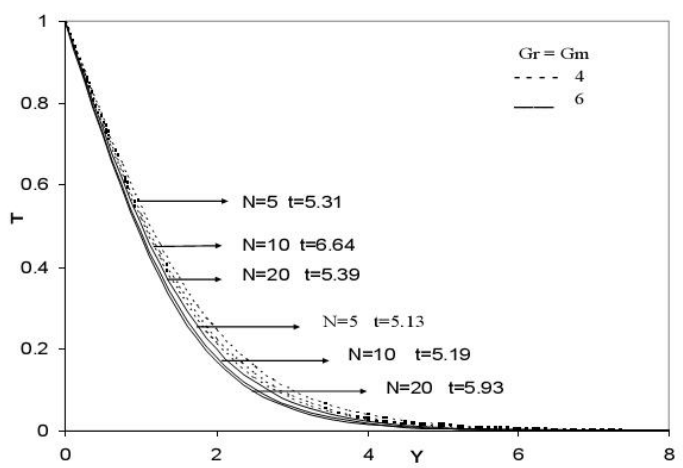

Fig. 11. Steady state temperature profiles at $\mathrm{X}=1.0$ for different $\mathrm{Gr}, \mathrm{Gm}$ and $\mathrm{N}$.

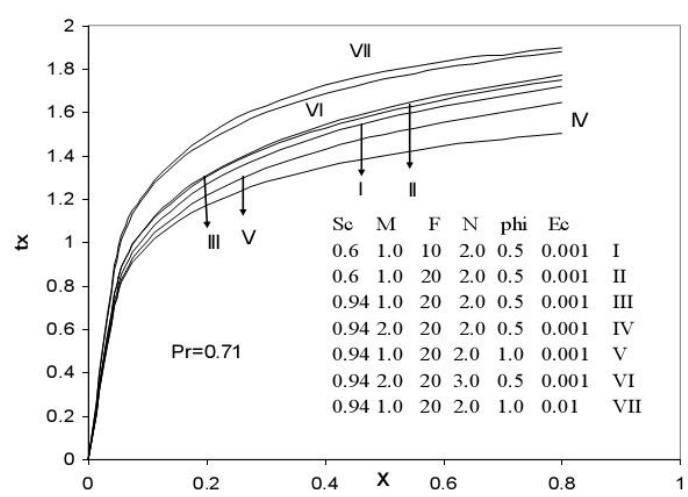

Fig. 12. Local skin friction. 
S. Suneetha et al. / JAFM, Vol. 4, No. 1, pp. 107-113, 2011.

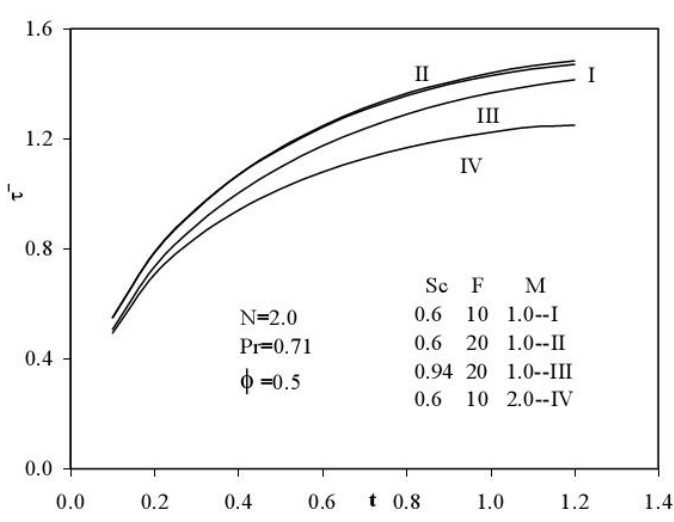

Fig. 13. Average skin friction.

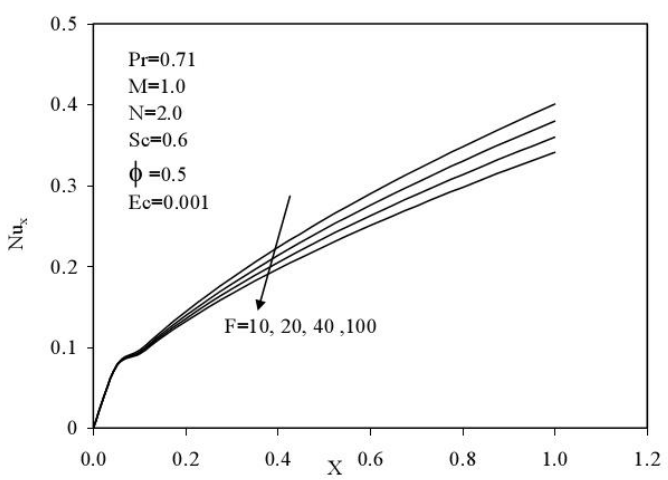

Fig. 14. Local Nusselt number.

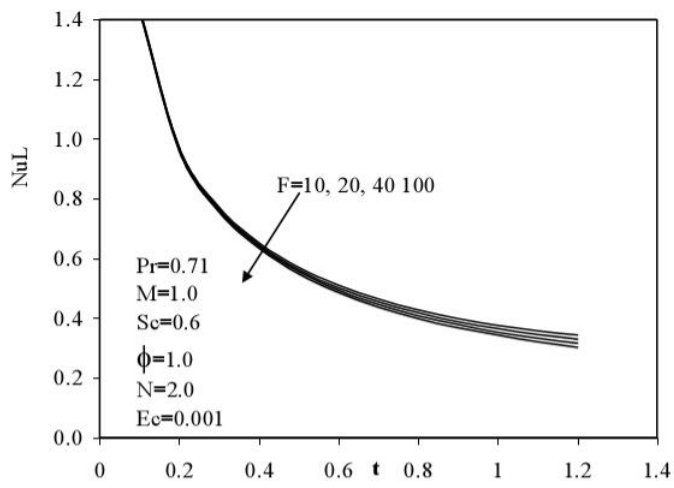

Fig. 15. Average Nusselt number.

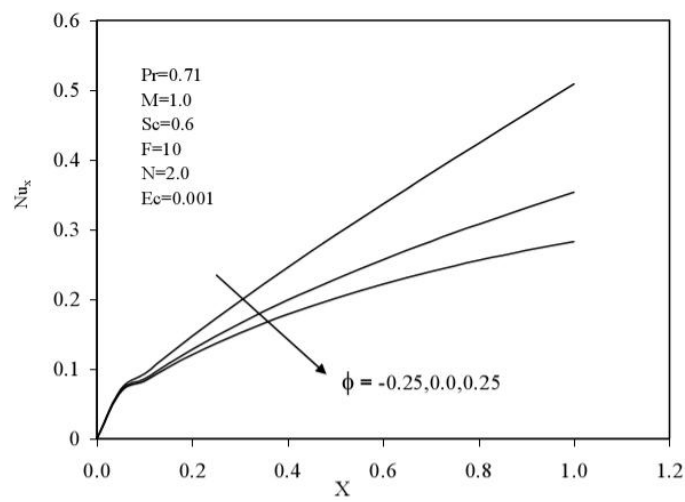

Fig. 16. Effect of $\varphi$ on local Nusselt number.

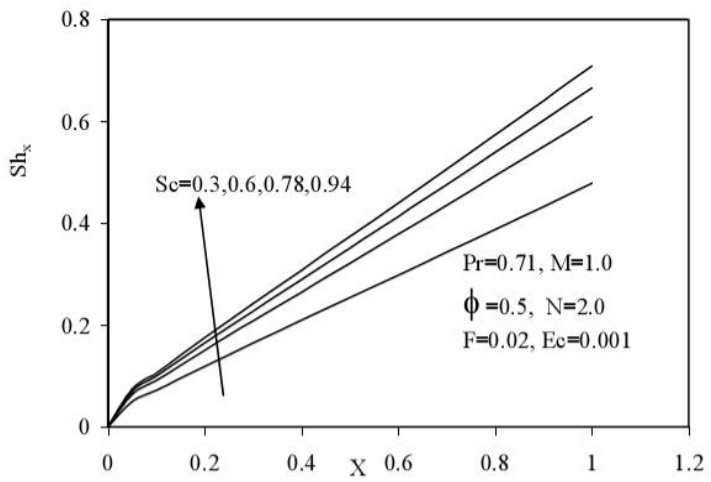

Fig. 17. Local Sherwood number.

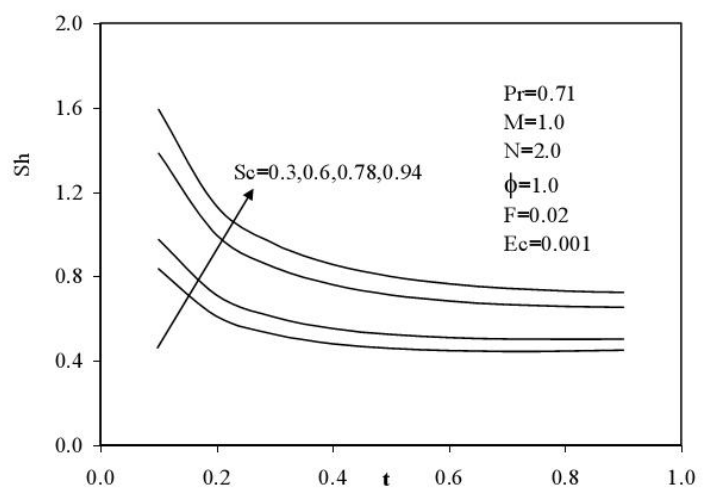

Fig. 18. Average Sherwood number. 